\title{
Case Study on Focal Retrograde Amnesia due to Limbic Encephalitis
}

\author{
Nobuhiro Takahashi',2, Mimpei Kawamura ${ }^{1,2}$, Naoki Tanihara ${ }^{2,3}$, \\ Mamiko Sato ${ }^{4}$, Yasutaka Kobayashi',2
}

\footnotetext{
${ }^{1}$ Department of Rehabilitation, Speech-Language-Hearing Therapy, Fukui Health Science University, Fukui, Japan

${ }^{2}$ Fukui Higher Brain Dysfunction Support Center, Fukui, Japan

${ }^{3}$ Department of Rehabilitation, Speech-Language-Hearing Therapy, Fukui General Hospital, Fukui, Japan

${ }^{4}$ Department of Rehabilitation Medicine, Fukui General Hospital, Fukui, Japan

Email: fchs-takahashi@kxb.biglobe.ne.jp
}

How to cite this paper: Takahashi, N., Kawamura, M., Tanihara, N., Sato, M. and Kobayashi, Y. (2020) Case Study on Focal Retrograde Amnesia due to Limbic Encephalitis. World Journal of Neuroscience, 10, $91-100$.

https://doi.org/10.4236/wjns.2020.102010

Received: February 15, 2020

Accepted: April 7, 2020

Published: April 10, 2020

Copyright $\odot 2020$ by author(s) and Scientific Research Publishing Inc. This work is licensed under the Creative Commons Attribution International License (CC BY 4.0).

http://creativecommons.org/licenses/by/4.0/

\section{(c) (i) Open Access}

\begin{abstract}
This case study addresses episodic memory impairment or focal retrograde amnesia related to limbic encephalitis. The patient in question was a right-handed man in his 60 s who developed focal retrograde amnesia due to limbic encephalitis. An abnormal MRI signal was observed in the right medial temporal lobe. Immediately after the encephalitis onset, mental symptoms-including impaired consciousness, disorientation, hallucinations, and delusions-appeared; however, his condition improved with medical treatment. After transfer to a convalescent and rehabilitation hospital, various neuropsychological tests were conducted but no apparent functional decline was observed in intellectual function, attention, memorization, or retention. However, the patient exhibited impairments related to autobiographical memory and memory of events $4-5$ years before the onset of limbic encephalitis. Based on these observations, the patient was considered to exhibit focal retrograde amnesia due to limbic encephalitis. Despite exhibiting retrograde amnesia, he had no difficulty in his daily life in the ward. Moreover, after discharge from hospital, smooth work reinstatement was possible. This case demonstrates that, even after exhibiting retrograde amnesia, if its duration is relatively short and physical function and other higher-order brain functions are sustained, social rehabilitation-including work reinstatement-is feasible.
\end{abstract}

\section{Keywords}

Limbic Encephalitis, Focal Retrograde Amnesia, Medial Temporal Lobe, Topographic Disorientation, Social Rehabilitation 


\section{Introduction}

Episodic memory impairment is classified as anterograde or retrograde amnesia in accordance with the time of onset. Anterograde amnesia refers to amnesia involving events after the onset. In contrast, retrograde amnesia refers to failure to recall memories of events from before the onset. The medial temporal lobe is a typical area where a lesion after brain injury causes episodic memory impairment. Following the H.M. case report [1], it was elucidated that the medial temporal lobe is an important neural structure related to episodic memory and that patients with injury to this region may exhibit both anterograde and retrograde amnesia. Moreover, patients with anterograde amnesia above a certain level of severity tend to also exhibit retrograde amnesia [2]. In 1980, Roman-Campos [3] reported a case where the patient exhibited only retrograde amnesia and no anterograde amnesia; since then, similar cases have rarely been reported. Retrograde amnesia without the accompanying anterograde amnesia is known as focal retrograde amnesia and illustrates the importance of understanding the neurological basis of episodic memory. Thus, reports on such cases are valuable. Moreover, higher-order brain function impairment, such as memory impairment, becomes a serious obstacle to social rehabilitation, including work reinstatement [4]. To the best of our knowledge, there are no existing reports on social rehabilitation related to cases of focal retrograde amnesia.

This report addresses a case in which the patient exhibited focal retrograde amnesia as a result of limbic encephalitis and describes the head MRI findings, symptoms, neuropsychological test results, the mechanism of appearance of focal retrograde amnesia, and the feasibility of social rehabilitation.

\section{Case Report}

The patient in this report was a right-handed man in his $60 \mathrm{~s}$ who worked in the community collaboration section of a hospital. The patient provided written informed consent for this case report. On day 32 pre-hospitalization, the patient developed snoring in breathing and underwent examination in the emergency outpatient department; however, as no abnormalities could be verified, he was sent home. On day 19 pre-hospitalization, he fell unconscious temporarily at home but soon recovered and continued with his daily activities without any further problems. However, he was later admitted to an acute care hospital after systemic convulsions and consciousness impairment were observed. After hospital admission, no abnormalities could be verified using blood tests, a cerebrospinal fluid test, and head MRI scans, but consciousness impairment persisted. The patient was diagnosed with viral encephalitis and anticonvulsants and antiviral agents were administered. Steroid pulse therapy was started from day 15 post-hospitalization, but on day 19 post-hospitalization, systemic convulsions appeared again and disorientation, hallucinations, and delusions were observed. The anticonvulsant dosage was increased from that day. On the MRI scan taken on day 20 post-hospitalization, a T2WI/FLAIR high signal was observed in the 
medial temporal lobe, including the right hippocampus, right parahippocampal gyrus, and right amygdala, and the patient was diagnosed with limbic encephalitis (Figure 1(a)).

Moreover, on day 27 post-hospitalization, an increase in brain edema was observed on the head MRI scan (Figure 1(b)). The second course of steroid pulse therapy was started on day 29 post-hospitalization. On day 35 post-hospitalization, the MRI scan of the head showed a reduction trend in the swelling (Figure 1(c)). Thereafter, no convulsions were observed. No motor or sensory impairment in the limbs, trunk, or face was observed and the patient was able to walk on his own. It was observed that even though the hospital was the patient's place of work, he often lost his way while moving around the building. On day 73 post-hospitalization, the patient was transferred to a convalescent and rehabilitation hospital for

(a)

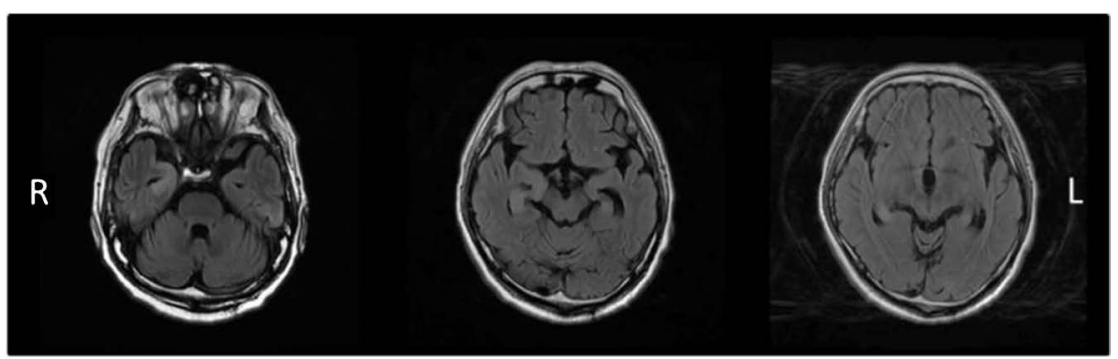

(b)

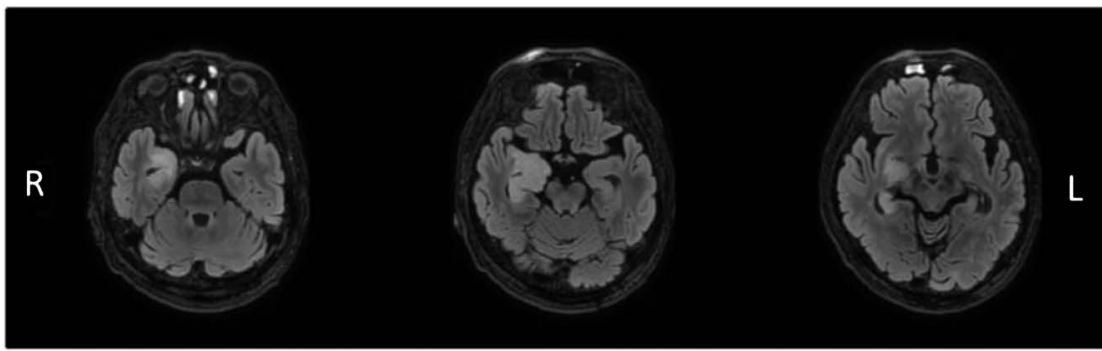

(c)

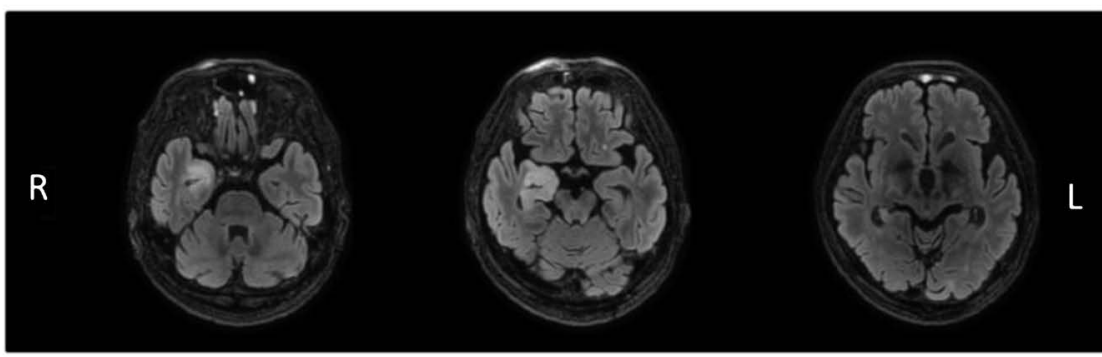

(d)

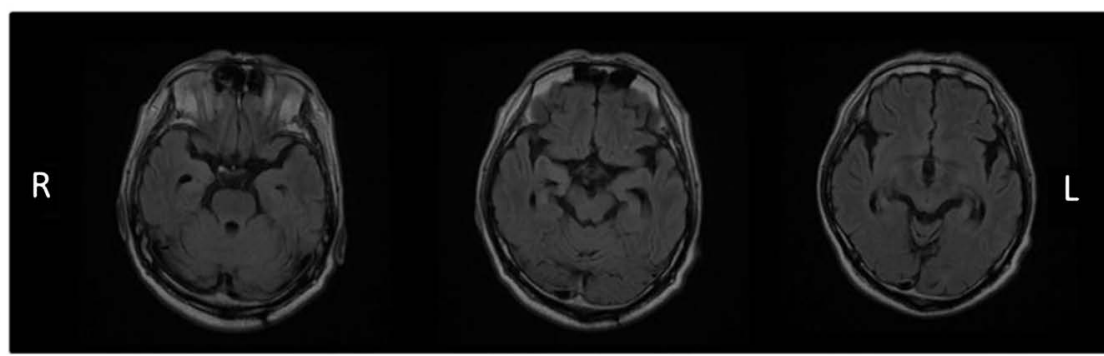

Figure 1. Changes in MRI FLAIR of the head: (a) day 20 post-hospitalization; (b) day 27 post-hospitalization; (c) day 35 post-hospitalization; and (d) day 91 post-hospitalization. 
rehabilitation for higher-order brain function impairment.

Neuropsychological tests were conducted (day $74-79$ post-hospitalization) from the day after his transfer to the rehabilitation hospital.

No decline in intellectual function was observed using Wechsler Adult Intelligence Scale, $3^{\text {rd }}$ edition (WAIS-III) [5]. The Wechsler Memory Scale-Revised (WMS-R) [6] scores were within the normal range and no memory impairment was observed in the Rivermead Behavioural Memory Test (RBMT) [7] results. In the Clinical Assessment for Attention (CAT) [8], the scores on the Memory Updating Test and the Paced Auditory Serial Addition Task (PASAT) were below the cut-off point; however, the other subtest scores reflecting basic attention were all within the normal range. In the Visual Perception Test for Agnosia (VPTA) [9], the patient had difficulty in sketching the layout of his own house. While sketching he said, "I feel that I have not been to my house for a long time. Actually, only the image of the house at the time when I got married comes up." Twenty days after the VPTA, when he was asked to sketch the layout of his own house, he was able to roughly sketch the layout correctly. However, regarding the kitchen, he sketched the old layout prior to the renovation (the kitchen had been renovated about three years before).

As topographic disorientation was suspected based on the VPTA results, topographic knowledge was evaluated again on day 86 post-hospitalization using the landscape image identification task and the verbal description of the route-to-destination task. In the landscape image identification, the evaluation was conducted using 30 images (old) of thought-to-be-known landscapes and 15 images (new) within the hospital that the patient saw for the first time after disease onset. Landscapes that had changed considerably during the five years prior to disease onset were excluded from the images of old landscapes. The patient correctly identified 29 of the 30 images of old landscapes and 14 of the 15 new images. In the verbal description of a route to destination, the evaluation was conducted using the route (old) from the patient's own house to his workplace, and a route (new) inside the hospital. In both cases, verbal descriptions were smoothly presented.

The symptom of losing his way during his hospitalization in the acute care hospital disappeared quickly after transfer to the rehabilitation hospital. The patient was also able to memorize the appearance and names of hospital staff. Moreover, the patient could easily memorize schedules such as rehabilitation times and upcoming plans. On the MRI scan taken on day 91 post-hospitalization, only a very weak abnormal signal was observed in the right medial temporal lobe (Figure 1(d)). Table 1 shows the neuropsychological test results.

On day 98 - 99 post-hospitalization, a trial overnight stay outside the hospital was undertaken. For this stay, the patient was asked to sit on the passenger seat and attempt to assist the family member driver with the navigation from the hospital to his own house. The results indicated that navigation was correctly provided. The patient also did not lose his way when moving around his neighborhood. 
Table 1. Neuropsychological test results.

\begin{tabular}{|c|c|c|c|}
\hline Neuropsychological test & & Current case & Normal range \\
\hline \multicolumn{4}{|l|}{ WAIS-III } \\
\hline Verbal IQ & & 92 & $100(15)^{\star}$ \\
\hline Performance IQ & & 99 & \\
\hline Full-Scale IQ & & 95 & \\
\hline Verbal Comprehension Index & & 93 & \\
\hline Perceptual Organization Index & & 119 & \\
\hline Working Memory Index & & 102 & \\
\hline Processing Speed Index & & 100 & \\
\hline \multicolumn{4}{|l|}{ WMS-R } \\
\hline Verbal Memory Index & & 93 & $100(15)^{*}$ \\
\hline Visual Memory Index & & 97 & \\
\hline General Memory Index & & 93 & \\
\hline Attention/Concentration Index & & 103 & \\
\hline Delayed Recall Index & & 93 & \\
\hline \multicolumn{4}{|l|}{ RBMT } \\
\hline Standardized Profile Score & & $23 / 24$ & $>15^{\star *}$ \\
\hline Screening Score & & $11 / 12$ & $>5^{* *}$ \\
\hline \multicolumn{4}{|l|}{ CAT } \\
\hline \multirow[t]{2}{*}{ Digit Span } & Forward & 5 & $>4^{* *}$ \\
\hline & Backward & 4 & $>3^{* *}$ \\
\hline \multirow[t]{2}{*}{ Tapping Span } & Forward & 5 & $>4^{* *}$ \\
\hline & Backward & 7 & $>4^{* *}$ \\
\hline \multicolumn{4}{|l|}{ Visual Cancellation Task } \\
\hline \multirow[t]{4}{*}{ Correct answer rate (\%) } & Task 1 & 98.2 & $97.5(2.2)^{\star}$ \\
\hline & Task 2 & 100.0 & $99.5(1.0)^{*}$ \\
\hline & Task 3 & 99.1 & $99.1(1.1)^{*}$ \\
\hline & Task 4 & 99.1 & $96.1(3.2)^{*}$ \\
\hline \multicolumn{4}{|l|}{ Auditory Detection Task } \\
\hline Correct answer rate (\%) & & 96.0 & $>94^{* *}$ \\
\hline \multicolumn{4}{|l|}{ Symbol Digit Modalities Test } \\
\hline Completion rate (\%) & & 44.5 & $>30^{* *}$ \\
\hline \multicolumn{4}{|l|}{ Memory updating Test } \\
\hline \multirow[t]{2}{*}{ Correct answer rate $(\%)$} & 3 span & 43.8 & $>56^{* *}$ \\
\hline & 4 span & 37.5 & $>38^{* *}$ \\
\hline \multicolumn{4}{|c|}{ Paced Auditory Serial Addition Test } \\
\hline \multirow[t]{2}{*}{ Correct answer rate $(\%)$} & 2 second & 20.0 & $>38^{* *}$ \\
\hline & 1 second & 3.3 & $>23^{* *}$ \\
\hline \multicolumn{4}{|l|}{ Position Stroop Test } \\
\hline Completion rate (\%) & & 100.0 & $>96^{* *}$ \\
\hline
\end{tabular}

Abbreviations: IQ, intelligence quotient; WAIS-III, Wechsler Adult Intelligence Scale, $3^{\text {rd }}$. edition; WMS-R, Wechsler Memory Scale-Revised; RBMT, Rivermead Behavioural Memory Test; CAT, Clinical Assessment for Attention. ${ }^{\star}$ Mean (1 standard deviation); ${ }^{* *}>$ Cut-off point. 
Moreover, inside his house, while he felt a little uncomfortable with the positioning of the furniture, there was no such feeling regarding the layout of the rooms. While riding in the car used for the trial overnight stay, he looked at the surrounding landscape from the car window and remarked, "I do not know this place." This was the route often taken by the patient before disease onset (note that the route was opened about five years before disease onset).

An evaluation of the patient's retrograde amnesia was performed on day 104 post-hospitalization using social events and autobiographical memories. Considering social events, the patient was asked whether he knew about representative domestic and international events that had occurred within the last five years. The number of correct responses for each year were, 0 out of $9(0 \%)$ for the year of encephalitis onset, 1 out of $9(11.1 \%)$ for one year prior, 0 out of $3(0 \%)$ for two years prior, 2 out of 5 (40\%) for three years prior, 5 out of $6(83.3 \%)$ for four years prior, and 5 out of 5 (100\%) for five years prior. The results suggested that the patient's memory for social events 3 - 4 years prior to disease onset was either lost or vague. Table 2 shows the questions and the patient's responses.

Considering autobiographical memories, the memories of the patient's own work content in the four years prior to disease onset were vague; specifically, the memories of the last year prior to disease onset were completely lost. Moreover, he travelled with his family every year during the five years before disease onset, but all such memories were lost.

On day 111 post-hospitalization, the patient was discharged from hospital to home-based care and started outpatient rehabilitation. After reinstatement at work, he was able to commute using public transport. He was also able to move around the workplace without losing his way. However, he was still unable to recall the content of his work one year prior to disease onset. As it was still difficult for him to drive a car, his role was changed to the task of mainly distributing public relations materials and he was able to smoothly familiarize himself with the new work. On day 135 post-hospitalization, it was concluded that he had no difficulty in carrying out his activities of daily living or his duties at work, and the outpatient rehabilitation intervention was terminated.

Table 2. Social event questions and patient's responses.

\begin{tabular}{|c|c|c|}
\hline Year & Social event & Reaction \\
\hline $\begin{array}{l}2017 \text { (Disease } \\
\text { onset) }\end{array}$ & $\begin{array}{c}\text { Donald Trump became President of the } \\
\text { United States }\end{array}$ & $\begin{array}{l}\text { Is this person the child of a former } \\
\text { president, or his wife? }\end{array}$ \\
\hline 2016 & Kumamoto earthquake occurred & Did such a thing happen? \\
\hline 2015 & Hokuriku Shinkansen opened & It was under construction, but did it open? \\
\hline 2014 & Consumption tax increased to $8 \%$ & Is it not $5 \% ?$ \\
\hline 2013 & $\begin{array}{c}\text { Mt. Fuji registered as a World Cultural } \\
\text { Heritage Site }\end{array}$ & I know. \\
\hline 2012 & London Olympics held & I know. \\
\hline
\end{tabular}




\section{Discussion}

In the current case, the patient showed no major episodic memory impairment concerning daily living after transfer to the convalescent and rehabilitation hospital. He was able to memorize the appearances and names of hospital staff, schedules, and upcoming plans; after discharge from the hospital, he was able to learn his new job description at work. Considering the WMS-R and RBMT scores, memorization and retention abilities were within the normal range. Accordingly, it was considered that anterograde amnesia was not present, and even if present, it was minor. However, autobiographical memories and memories of social events during the 4 - 5 years prior to the onset of limbic encephalitis were vague, indicating retrograde amnesia. These symptoms concur with the diagnosis focal retrograde amnesia [3].

In previous reports, it was shown that relatively short-term focal retrograde amnesia cases were often caused by encephalitis [10]-[15]. In addition, in the initial stage, abnormalities in the medial temporal lobe can be verified through MRI results and other means; however, after focal retrograde amnesia has been firmly established, abnormalities cannot be clearly verified using MRI [10]. These reports are in agreement with the observations in the present case that relatively short-term focal retrograde amnesia appeared due to encephalitis and that the MRI scan showed a very weak abnormality signal when only retrograde amnesia persisted. Sehm et al. [16] compared a group of healthy individuals with a case of focal retrograde amnesia after an epileptic seizure where a lesion could not be clearly identified on MRI scans. They concluded that gray matter decreases in the left temporopolar cortex and a region between the right parahippocampal gyrus and the lingual cortex was associated with focal retrograde amnesia. In the present case, on the MRI scan taken in the initial stage after disease onset, signal abnormalities were detected in the medial temporal lobe including the right hippocampus, right parahippocampal gyrus, and right amygdala. It is thought that even without clear instrumental abnormalities being detected with MRI, the medial temporal lobe-including regions such as the right parahippocampal gyrus-did have persistent functional deficits, leading to the persistence of retrograde amnesia.

In the present case, regarding the retrograde amnesia for the period of 4 - 5 years before disease onset, the most recent memories showed the maximum impairment, and further into the past, the amount of information that could not be recalled and the inaccuracies decreased. This is consistent with the "temporal gradient" of retrograde amnesia, which has been explained using the concept of memory consolidation [17] [18]. The contents of the most recent memory, which has not yet completed the process of consolidation, are most prone to impairment, while the contents of memory for which there has been enough time to complete the process of consolidation do not suffer impairment. The medial temporal lobe is responsible for memory consolidation and as the contents of memory for which consolidation is completed are kept in the neocortex, 
damage to the medial temporal lobe can cause retrograde amnesia with a temporal gradient. Squire et al. [19] report that for systems consolidation-which is a part of memory consolidation-in the initial stage, the memory is stored in both the medial temporal lobe and neocortex, centered around the hippocampus; thereafter, the hippocampus gradually reorganizes the information in the neocortex and stabilizes it, ultimately making it independent from the hippocampus. In the present case, the observations regarding the presence of temporal gradient in memory, the changes in the medial temporal lobe lesion, and the fact that the cerebral cortex area was immune to inflammation support the systems consolidation theory proposed by Squire et al. [19].

In the present case, the patient often lost his way inside the acute care hospital, which was also his workplace, and topographic disorientation was suspected. On the MRI scan in acute care, distinct abnormal signals were detected in the medial temporal lobe. The right parahippocampal gyrus is considered to be important regarding lesions in streetscape agnosia, which refers to impairment in the identification of familiar buildings and landscapes [20] [21]. In the present case, distinct abnormal signals in the right parahippocampal gyrus were detected on the acute care MRI, and it is likely that streetscape agnosia appeared at this time. However, after the patient's transfer to the convalescent and rehabilitation hospital, his tendency of losing his way both inside the hospital and around his own neighborhood disappeared and he was able to perform navigation while riding in a car. Thereafter, in the evaluation of topographic knowledge, he was able to identify old and new landscapes as well as describe the route to a destination. This suggests that after his transfer to the convalescent and rehabilitation hospital, the topographic disorientation may have mostly disappeared. However, during the VPTA conducted at this time, he had difficulty in sketching the layout of his own house. Moreover, during the trial overnight stay, when taking the route he had often used before disease onset, he reported not knowing it. These two points are observations that points toward topographic disorientation; however, it is necessary to consider the period of retrograde amnesia in this case study, which is about $4-5$ years. Considering that the patient could not recall the images of landscapes that had undergone topographic changes during this period, it is possible to explain these observations as symptoms of focal retrograde amnesia.

In this case study, the patient was able to return to work after discharge from hospital, and despite changes in the content of his work, he was able to learn the new tasks. This case study suggests that social rehabilitation-including reinstatement at work-is feasible when there is no impairment in higher-order brain functions and no physical function abnormalities, even if focal retrograde amnesia persists for a relatively short period. Although focal retrograde amnesia is a disorder that can result in the loss of personal identity, it is inferred that it may not be an obstacle to social rehabilitation. To the best of our knowledge, there are no previous reports on social rehabilitation in cases of focal retrograde am- 
nesia, and it is hoped that more of such cases will be reported in the future.

\section{Conclusion}

In this case study, we report on a patient who exhibited retrograde amnesia involving memory spanning a period of 4 - 5 years as a result of limbic encephalitis. Anterograde amnesia was not observed, indicating that the patient exhibited focal retrograde amnesia. The focal retrograde amnesia in this case study was thought to be associated with the functional decline of the right medial temporal lobe due to limbic encephalitis. The patient had no difficulty in his daily life in the hospital and smooth work reinstatement was possible after discharge. The results of this case suggest that social rehabilitation-including reinstatement at work-may be feasible in cases of focal retrograde amnesia when physical functions and higher-order brain functions are sustained after developing retrograde amnesia for a relatively short period.

\section{Conflicts of Interest}

The authors declare no conflicts of interest regarding the publication of this paper.

\section{References}

[1] Scoville, W.B. and Milner, B. (1957) Loss of Recent Memory after Bilateral Hippocampal Lesions. Journal of Neurology, Neurosurgery, and Psychiatry, 20, 11-21. https://doi.org/10.1136/jnnp.20.1.11

[2] Smith, C.N., Frascino, J.C., Hopkins, R.O. and Squire, L.R. (2013) The Nature of Anterograde and Retrograde Memory Impairment after Damage to the Medial Temporal Lobe. Neuropsychologia, 51, 2709-2714. https://doi.org/10.1016/j.neuropsychologia.2013.09.015

[3] Roman-Campos, G., Poser, C.M. and Wood, F.B. (1980) Persistent Retrograde Memory Deficit after Transient Global Amnesia. Cortex, 16, 509-518. https://doi.org/10.1016/S0010-9452(80)80053-0

[4] Senzaki, A. (2017) Return to Work after Brain Injury in Japan. Japan Journal of Rehabilitation Medicine, 54, 270-273. https://doi.org/10.2490/jjrmc.54.270

[5] Fujita, K., Maekawa, H., Dairoku, K. and Yamanaka, K. (2007) The Japanese Version of the Wechsler Adult Intelligence Scale-III. Nihon Bunka Kagakusha, Tokyo.

[6] Sugishita, M. (2007) The Japanese Version of the Wechsler Memory Scale-Revised. Nihon Bunka Kagakusha, Tokyo.

[7] Watamori, T., Hara, H., Miyamori, T. and Eto, F. (2002) The Japanese Version of the Rivermead Behavioural Memory Test. Chiba Test Center, Tokyo.

[8] Japan Society for Higher Brain Dysfunction (2008) Clinical Assessment for Attention. Shinko Igaku Syuppansya, Tokyo.

[9] Japan Society for Higher Brain Dysfunction (2003) Visual Perception Test for Agnosia. Shinko Igaku Syuppansya, Tokyo.

[10] Hokkanen, L., Launes, J., Vataja, R., Valanne, L. and Iivanainen, M. (1995) Isolated Retrograde Amnesia for Autobiographical Material Associated with Acute Left Temporal Lobe Encephalitis. Psychological Medicine, 25, 203-208. 
https://doi.org/10.1017/S0033291700028245

[11] Yoneda, Y., Yamadori, A., Mori, E. and Yamashita, H. (1992) Isolated Prolonged Retrograde Amnesia. European Neurology, 32, 340-342. https://doi.org/10.1159/000116857

[12] Yamadori, A., Yoneda, Y., Mori, E. and Yamashita, H. (1994) Amnesia Due to Bilateral Hippocampal Lesions. Advances in Neurological Sciences, 38, 997-1003.

[13] Ishihara, K., Ichikawa, H., Takeuchi, T., Kawamura, M. and Sugita, K. (1997) Isolated Retrograde Amnesia Following Viral Encephalitis. Rinsho Shinkeigaku (Clinical Neurology), 37, 509-513.

[14] Ikeda, M. (1998) Retrograde Amnesia. Higher Brain Function Research, 18, 189-195. https://doi.org/10.2496/apr.18.189

[15] Yamadori, A., Suzuki, K., Shimada, M., Tsukiura, T., Morishima, T. and Fujii, T. (2001) Isolated and Focal Retrograde Amnesia: A Hiatus in the Past. Tohoku Journal of Experimental Medicine, 193, 57-65. https://doi.org/10.1620/tjem.193.57

[16] Sehm, B., Frisch, S., Thöne-Otto, A., et al. (2011) Focal Retrograde Amnesia: Voxel-Based Morphometry Findings in a Case without MRI Lesions. PLOS ONE, 6, e26538. https://doi.org/10.1371/journal.pone.0026538

[17] Fujii, T., Moscovitch, M. and Nadel, L. (2000) Memory Consolidation, Retrograde Amnesia and the Temporal Lobe. In: Boller, F. and Grafman, J., Eds., Handbook of Neuropsychology, 2nd Edition, Vol. 2, Elsevier Science BV, New York, 223-250.

[18] Brown, A.S. (2002) Consolidation Theory and Retrograde Amnesia in Humans. Psychological Bulletin Review, 9, 403-425. https://doi.org/10.3758/BF03196300

[19] Squire, L.R., Genzel, L., Wixted, J.T. and Morris, R.G. (2015) Memory Consolidation. Cold Spring Harbour Perspectives in Biology, 7, a021766.

https://doi.org/10.1101/cshperspect.a021766

[20] Takahashi, N. and Kawamura, M. (2002) Pure Topographical Disorientation: The Anatomical Basis of Topographical Agnosia. Cortex, 38, 717-725. https://doi.org/10.1016/S0010-9452(08)70039-X

[21] Epstein, R. and Kanwisher, N. (1998) A Cortical Representation of the Local Visual Environment. Nature, 392, 598-601. https://doi.org/10.1038/33402 